CLINICAL STUDY

\title{
Irisin mRNA and circulating levels in relation to other myokines in healthy and morbidly obese humans
}

\author{
Maria T Vamvini ${ }^{1}$, Konstantinos N Aronis ${ }^{1,2,3}$, Grigorios Panagiotou ${ }^{1}$, Joo Young Huh ${ }^{1}$, John P Chamberland ${ }^{1}$, \\ Mary T Brinkoetter ${ }^{1}$, Michael Petrou ${ }^{4}$, Costas A Christophi ${ }^{5}$, Stefanos N Kales ${ }^{6}$, David C Christiani ${ }^{6}$ and \\ Christos S Mantzoros ${ }^{1,3}$ \\ ${ }^{1}$ Division of Endocrinology, Diabetes and Metabolism, Harvard Medical School, Beth Israel Deaconess Medical Center, 330 Brookline Avenue, Boston, \\ Massachusetts 02215, USA, ${ }^{2}$ Department of Internal Medicine, Boston Medical Center, Boston University, Boston, Massachusetts, USA, ${ }^{3}$ Section of \\ Endocrinology, Boston VA Healthcare System, Boston, Massachusetts, USA, ${ }^{4}$ Cyprus Anti-Doping Authority, Nicosia, Cyprus, ${ }^{5}$ Cyprus International \\ Institute for Environmental and Public Health in Association with Harvard School of Public Health, Cyprus University of Technology, Limassol, Cyprus and \\ ${ }^{6}$ Department of Environmental Health, Harvard School of Public Health, Boston, Massachusetts, USA
}

(Correspondence should be addressed to C S Mantzoros; Email: cmantzor@bidmc.harvard.edu)

\begin{abstract}
Objective: Skeletal muscle is considered to be an endocrine organ that secretes a number of myokines including follistatin (FST), myostatin (MSTN), activin A, and the newly identified irisin. Irisin's biology and function exhibit similarities with the functions of the FST-MSTN-activin A axis. It remains unknown whether there is any interplay among these molecules. The aim of this study is to examine potential associations of irisin with the FST, MSTN, and activin A axis.

Methods: Two observational studies were performed to evaluate the associations of irisin with the other three peptides. Study A included 150 healthy males aged $18.48 \pm 0.16$ years with BMI $23.18 \pm 3.75 \mathrm{~kg} / \mathrm{m}^{2}$. Fasting serum samples were used to measure the levels of the molecules of interest. Study B included 14 morbidly obese individuals, candidates for bariatric surgery, aged $53.14 \pm 8.93$ years with BMI $50.18 \pm 10.63 \mathrm{~kg} / \mathrm{m}^{2}$. Blood samples were obtained after an overnight fast. Eight out of the 14 participants consented to an optional thigh biopsy during their bariatric surgery. Using the above blood and tissue samples, we measured circulating levels and muscle mRNA of irisin, FST, MSTN, and activin A.

Results: We report that FNDC5 mRNA in muscle is positively correlated with FST mRNA expression in morbidly obese subjects $(\rho=0.93, P<0.001)$. We also found that circulating irisin is positively correlated with FST circulating levels among lean subjects $(\rho=0.17, P=0.05)$ while this association was suggestive among the obese $(\rho=0.56, P=0.07)$.

Conclusion: The newly identified myokine irisin may be positively associated with FST at both the mRNA and circulating protein level.
\end{abstract}

European Journal of Endocrinology 169 829-834

\section{Introduction}

Recent studies have shed light on the role of nontraditional endocrine tissues, including skeletal muscle tissue, in the regulation of energy metabolism, body composition, and insulin sensitivity (1). A number of cytokines and other peptides, also known as myokines, are expressed and released by muscle fibers in response to contraction, differentiation, and insulin resistance (2). Irisin, the most recently identified myokine, is the extracellular cleaved product of fibronectin type III domain, containing 5 (FNDC5), and is regulated by peroxisome proliferator-activated receptor gamma coactivator $1-\alpha$ (PPARGC1A or PGC1 $\alpha$ ) (3). Studies in mice have shown that FNDC5/irisin overexpression induces browning and enhances thermogenesis of white adipose tissue, contributing to the improvement of glucose homeostasis and insulin resistance $(3,4)$. Our team recently studied the physiology of irisin in humans and reported a significant increase in irisin levels after acute exercise and significant association of its levels with anthropometric and metabolic parameters (5). In addition, two recently published studies demonstrated altered irisin levels in type 2 diabetes $(6,7)$ and in severely obese humans (8).

Despite the increased interest of the scientific community in studying the new myokine irisin (9), its physiology remains largely unknown. Irisin is one of the many identified myokines, but its association and/or interaction with other myokines remains unknown. Interestingly, irisin and follistatin (FST), a peptide that regulates muscle growth through the inhibition of myostatin (MSTN) and activin A, exhibit a similar response to exercise (10). Conversely, MSTN, a strong negative muscle mass regulator, changes toward the opposite direction after long-term aerobic exercise 
$(10,11)$. The potential interplay between MSTN and irisin was highlighted by Shan et al. (12) who reported that within the muscle tissue, $\operatorname{MSTN}(-/-)$ leads to increased $5^{\prime}$ AMP-activated protein kinase (AMPK) expression and phosphorylation, which subsequently activates irisin precursors PGC1 $\alpha$ and FNDC5.

The individual role of FST, MSTN, and activin A has been parsed out through in vitro and in vivo animal and human studies $(13,14,15)$. These molecules exhibit different responses to altered metabolism states, such as chronic energy deprivation, through a leptin-independent pathway (13). Their pivotal role in muscle mass regulation and in the muscle-adipose tissue cross-talk has already been well elucidated $(14,15)$. On the other hand, the pathophysiological role of irisin is currently being examined with both in vitro and in vivo studies. The apparent similarities of the above peptides in their response to exercise and their impact on body metabolism triggered our interest to investigate further whether there is cross-talk or associations at the mRNA or circulating protein levels between the novel myokine, irisin, and the FST-MSTN-activin A axis. The study herein aims to examine cross sectionally correlations among the circulating and tissue expression levels of irisin, FST, activin A, and MSTN in otherwise healthy subjects with a wide variety in BMI, from normal to morbidly obese.

\section{Subjects and methods}

\section{Cyprus Metabolism Study: cross-sectional study of healthy young males}

As described before, the Cyprus Metabolism Study included a total of 1056 healthy, 18-year-old men who were screened and enrolled in the Cyprus Metabolism Study at the beginning of their 2-year mandatory military service in the Cypriot Army (16). The participants were exposed to the same dietary, exercise, and sleep conditions for 1 day as they had commenced their mandatory military service the previous day. They were eligible for enrollment to this cohort as part of their health screening examination. Serum measurements were performed in all participants in a standardized manner after an overnight fast. This study has been approved by the Harvard School of Public Health Institutional Review Board and the Cyprus National Bioethics Committee and received an exempt status by the Institutional Review Board of Beth Israel Deaconess Medical Center (BIDMC).

To evaluate for any association between serum levels of irisin, FST, activin A, and MSTN, we randomly selected a sample of 150 participants of the Cyprus Metabolism Study. The randomization was performed using random subject identities, generated from the statistical software described below. The mean age of our sample was $18.48 \pm 0.16$ years and the mean BMI was $23.18 \pm 3.75 \mathrm{~kg} / \mathrm{m}^{2}$. There was not clinically significant difference between the baseline clinical characteristics of the total study population (1056 participants) and the 150 participants who were randomly selected for the purposes of this study (Supplementary Table 1, see section on supplementary data given at the end of this article).

\section{Cross sectional study of morbidly obese subjects}

Subjects A total of 14 morbidly obese subjects (age $53.14 \pm 8.92$ years, BMI $50.18 \pm 10.62 \mathrm{~kg} / \mathrm{m}^{2}$, males/ females $=8 / 6$ ) were recruited to this study after they were approved for bariatric surgery. All participants were between 18 and 65 years old and fulfilled the NIH criteria for bariatric surgery. The study was approved by the Institutional Review Board of BIDMC and participants provided written, informed consent to participate (5).

Study protocol Participants were examined by study physicians before surgery in the BIDMC General Clinical Research Center (GCRC). Blood samples were obtained after an overnight fast before surgery. Eight out of the fourteen participants consented to undergo an optional left or right thigh biopsy during the surgery. The biopsy was performed by the general surgeon and thigh skeletal muscle tissue was obtained using a biopsy needle (5).

\section{Measurement of circulating levels of irisin, FST, activin A, and MSTN}

The molecules of interest were measured with commercially available immunoassays. Irisin levels were determined using ELISA assay from Aviscera Biosciences (Santa Clara, CA, USA). Activin A and FST serum levels were determined using ELISA assay from R\&D Systems (Minneapolis, MN, USA). MSTN was tested with a commercially available competitive immunoassay (EIA by Immundiagnostik AG, Bensheim, Germany) (13, 17). All samples were analyzed in duplicate. Samples that yielded a coefficient of variability $>15 \%$ were excluded from the analysis.

\section{Measurement of PGC1 $\alpha$, FNDC5, PPARG, FST, activin $A$, and MSTN expressions in the skeletal muscle tissue}

PCG1 $\alpha$, FNDC5, PPARG, FST, activin A (INHBA), and MSTN expression was detected with real-time quantitative PCR (RT-qPCR). RNA was extracted from the skeletal muscle tissue with Trizol (Invitrogen), and first-strand cDNA synthesis was performed using the High Capacity cDNA Reverse Transcription Kit (Applied Biosystems), according to the manufacturer's protocol. For RT-qPCR, $10 \mathrm{ng}$ cDNA per $20 \mu \mathrm{l}$ reaction were 
Table 1 Baseline clinical and laboratory characteristics $(n=150)$.

\begin{tabular}{lcr}
\hline Continuous variables & Mean & \multicolumn{1}{c}{ s.D. } \\
\hline Age (years) & 18.48 & 0.16 \\
Weight $(\mathrm{kg})$ & 70.99 & 13.65 \\
Waist $(\mathrm{cm})$ & 81.16 & 10.46 \\
Waist:hip ratio & 0.84 & 0.05 \\
BMl $\left(\mathrm{kg} / \mathrm{m}^{2}\right)$ & 23.18 & 3.75 \\
Fat mass (\%) & 13.97 & 5.72 \\
Fat mass $(\mathrm{kg})$ & 10.61 & 6.65 \\
Fat-free mass $(\mathrm{kg})$ & 60.39 & 7.63 \\
BMR (kcal/day) & 1798.13 & 206.59 \\
Glucose (mg/dl) & 78.91 & 7.28 \\
Total cholesterol (mg/dl) & 146.94 & 27.64 \\
HDL (mg/dl) & 48.07 & 9.64 \\
LDL (mg/dl) & 103.34 & 25.19 \\
Triglycerides (mg/dl) & 59.6 & 23.12 \\
SAP (mmHg) & 107.22 & 11.05 \\
DAP (mmHg) & 61.84 & 8.09 \\
\hline
\end{tabular}

amplified using human-specific TaqMan Gene Expression Assay (Assay ID: Hs00246256 for FST, Hs00170103 for activin A, Hs00193363 for MSTN, Hs0106719 for PCG1 $\alpha$, Hs00401006 for FNDC5, Hs01115513 for PPARG, Applied Biosystems) in the 7500 Fast RT-qPCR system using standard realtime 7500 protocol. Data were analyzed using the 7500-system software (Applied Biosystems). mRNA expression was measured using the comparative $\mathrm{Ct}$ method (ABI7500 FAST, Applied Biosystems). Data were normalized to $\beta$-actin (id: Hs99999903) in each reaction (5).

\section{Statistical analysis}

Statistical analysis was performed with STATA version 12 (STATA Corp., College Station, TX, USA). Results are expressed as mean \pm s.D. for normally distributed variables. Normality of the dependent variables was evaluated using frequency histograms and the Shapiro-Wilk test. Variables that were not normally distributed were normalized with square-root or logarithmic transformation as appropriate. Correlations between different myokines were evaluated using Spearman's correlation analysis. One extreme outlier in irisin levels ( $>6$ s.D.) was removed from the analysis. All $P$ values reported herein are two-sided and alpha criterion is set to 0.05 .

\section{Results}

\section{Cyprus Metabolism Study: cross-sectional study results}

The baseline clinical and laboratory characteristics of the 150 participants are summarized in Table 1 . The mean age of the above participants was $18.48 \pm 0.16$ years and their mean BMI was $23.18 \pm 3.75 \mathrm{~kg} / \mathrm{m}^{2}$. There was only one participant whose parameters fulfilled the criteria for the diagnosis of metabolic syndrome (data not shown). Seven participants were on a multivitamin supplement, one on paracetamol when needed, two on inhalers for asthma, and one on valproate. None of the participants was on more than one medication.

On our correlation analysis, we found that circulating irisin levels are positively correlated with FST circulating levels $(\rho=0.17, P=0.05)$. We also reported the expected negative correlation of FST with circulating MSTN levels $(\rho=-0.19, P=0.02)$. Table 2 summarizes the findings from the correlation analysis of the four novel peptides.

\section{Cross-sectional study of morbidly obese subjects}

Serum measurements We studied 14 morbidly obese subjects that were scheduled to undergo bariatric surgery. Their mean age was $53.14 \pm 8.93$ years and mean BMI was $50.18 \pm 10.63 \mathrm{~kg} / \mathrm{m}^{2}$. Again there was a positive correlation of irisin with circulating FST which tended to be significant $(\rho=0.56, P=0.07)$. This confirmed our important finding from the Cyprus cross-sectional study, albeit without statistically significant $P$ values, probably due to the small number of participants in this study (Table 3).

mRNA expression studies The correlation matrix analysis of the expression levels of FNDC5, PGC1 $\alpha$, PPARG, FST, activin A, and MSTN is summarized in Table 4. We found that FNDC5 mRNA expression is positively correlated with FST mRNA expression in morbidly obese subjects $(\rho=0.93, P<0.001)$. This finding comes in agreement with our findings from the correlation analysis of circulating serum levels. Our analysis did not detect any other significant association at tissue level.

\section{Discussion}

Skeletal muscle is now considered to be an endocrine organ that secretes a number of myokines including FST,

Table 2 Correlation matrix of novel myokines in young healthy males $(n=150)$.

\begin{tabular}{lccc}
\hline & $\begin{array}{c}\text { Irisin } \\
(\mathrm{ng} / \mathrm{ml})\end{array}$ & $\begin{array}{c}\text { Follistatin } \\
(\mathrm{pg} / \mathrm{ml})\end{array}$ & $\begin{array}{c}\text { Activin A } \\
(\mathrm{pg} / \mathrm{ml})\end{array}$ \\
\hline Follistatin $(\mathrm{pg} / \mathrm{ml})$ & & & \\
$\rho$ & 0.17 & & \\
$P$ & $0.05^{\star}$ & & \\
Activin A (pg/ml) & & & \\
$\rho$ & -0.05 & -0.13 & \\
$P$ & 0.52 & 0.12 & \\
Myostatin (ng/ml) & & & \\
$\rho$ & -0.12 & -0.19 & 0.1 \\
$P$ & 0.15 & $0.02^{*}$ & 0.25 \\
\hline
\end{tabular}

*, significant values. 
Table 3 Correlation matrix of novel myokines in morbidly obese males and females $(n=14)$.

\begin{tabular}{lrrr}
\hline & $\begin{array}{c}\text { Irisin } \\
(\mathrm{ng} / \mathrm{ml})\end{array}$ & $\begin{array}{c}\text { Follistatin } \\
(\mathrm{pg} / \mathrm{ml})\end{array}$ & $\begin{array}{c}\text { Activin A } \\
(\mathrm{pg} / \mathrm{ml})\end{array}$ \\
\hline Follistatin $(\mathrm{pg} / \mathrm{ml})$ & & & \\
$\rho$ & 0.56 & & \\
$P$ & 0.07 & & \\
Activin A (pg/ml) & & & \\
$\rho$ & 0.06 & 0.30 & \\
$P$ & 0.85 & 0.37 & \\
Myostatin $(\mathrm{ng} / \mathrm{ml})$ & & & \\
$\rho$ & -0.07 & -0.42 & -0.24 \\
$P$ & 0.87 & 0.29 & 0.57 \\
\hline
\end{tabular}

MSTN, activin A, and irisin. These molecules seem to play a vital role in orchestrating the function and metabolism of other important endocrine organs (18). Irisin is the most recently identified myokine and its pathophysiology remains largely unknown. There is recent evidence about its role in browning of fat tissue, exercise physiology, and its associations with metabolic and anthropometric parameters $(3,5,8)$. In line with the results from mice (3) and rats (19), irisin was reported to correlate with energy expenditure (EE) in postmenopausal women, but only in a subgroup in which EE was greater than the one predicted by muscle mass (20). However, Fain et al. (21) recently showed that exercise does not increase muscle FNDC5 protein nor mRNA expression in pigs, although the circulating levels were increased, suggesting that there might be different mechanisms of irisin production and secretion among species. Our in vitro models (22) have proposed a possible linkage of irisin with the hippocampus, implying that irisin may exert central effects in the fight against Alzheimer's disease (23). Yet, it still remains unknown whether any association between irisin and the members of the FST-activin A-MSTN axis indeed exists. In this study, using cross-sectional data from healthy and morbidly obese subjects, we found a significant correlation of circulating irisin with circulating FST levels. We replicated this finding by examining the associations of the mRNA expression patterns at skeletal muscle tissue and found that FNDC5 mRNA levels were positively associated with FST mRNA levels in muscle.

FST is a peptide that has been extensively studied and was initially considered to be a reproductive hormone due to its FSH inhibitory role (24). However, further studies have been suggestive of FST's multifunctional role. It is a potent MSTN and activin A inhibitor, regulating fat and lean mass and promoting muscle growth, glucose uptake, and insulin sensitivity $(14,25)$. Acute exercise increases FST levels shortly after a bout of acute exercise (10). It is interesting that irisin also responds to acute exercise in a very similar pattern with FST (5). This parallel response triggered our interest in studying the existence of any associations between these two molecular entities. In our study, we reported that there is indeed a positive correlation of FST with irisin both at the tissue level and in circulation. This association was confirmed by studying two different populations: young healthy males and morbidly obese males and females. The above finding may indicate the existence of direct interaction between the two myokines, with FST triggering the expression of irisin or vice versa or the presence of common underlying mechanisms regulating both peptides in a similar manner. Interestingly, our correlation analysis showed a significantly higher correlation of irisin with FST in the obese population $(\rho=0.56)$ compared with the healthy male population $(\rho=0.17)$, but larger studies are needed to confirm or refute this observation. Mechanisms underlying this association need to be further investigated in future studies. It is interesting to note that in our, rather limited in size, data set PGC1 $\alpha$ was not correlated with FNDC5 gene expression in muscle. Although $\mathrm{PGC} 1 \alpha$ has been suggested to be the upstream regulator of FNDC5, PGC1 $\alpha$ is apparently not the only modifier of irisin expression and production. It has been proposed, for example, that in states of nonexercise-related metabolism, such as at baseline in obese and insulin-resistant subjects, irisin/FNDC5 might be regulated by additional stimuli including myokines in a PGC1 $\alpha$-independent manner (26). It has also been proposed that in the obese state irisin may also be secreted by the adipose tissue $(26,27)$. This may explain why we failed to find a direct association of fat free mass with irisin levels in our analyses. The possible presence of other molecules serving as upstream inducer(s) or suppressors of irisin/FNDC5 needs to be extensively studied in the future.

MSTN has a powerful negative effect on myogenesis and glucose homeostasis (28), while activin A is a multifunctional protein, secreted by various tissues, that promotes pre-adipocyte proliferation, regulates inflammation processes, muscle homeostasis, and glucose-induced insulin secretion (29). Both MSTN

Table 4 Correlation matrix of mRNA relative expression of novel myokines in muscle in morbidly obese subjects $(n=8)$.

\begin{tabular}{lrrrrr}
\hline & FNDC5 & PGC1 $\alpha$ & PPARG & Follistatin & Activin A \\
\hline PGC1 $\alpha$ & & & & & \\
$\rho$ & 0.05 & & & & \\
$P$ & 0.91 & & & & \\
$P P A R G$ & & & & & \\
$\rho$ & -0.02 & 0.17 & & & \\
$P$ & 0.95 & 0.69 & & & \\
Follistatin & & & & & \\
$\rho$ & $0.93^{*}$ & 0.24 & 0.21 & & \\
$P$ & $<0.001$ & 0.57 & 0.61 & & \\
Activin A & & & & & \\
$\rho$ & 0.19 & 0.19 & 0.50 & 0.36 & \\
$P$ & 0.65 & 0.65 & 0.21 & 0.38 & \\
Myostatin & & & & & \\
$\rho$ & 0.36 & 0.24 & -0.26 & 0.43 & 0.45 \\
$P$ & 0.39 & 0.57 & 0.53 & 0.29 & \\
\hline
\end{tabular}


and activin A exert their function through the activin type II receptors $A$ and $B$ and share the same extracellular antagonist, i.e. FST (14). In contrast to irisin and FST, MSTN has been shown to decrease after exercise (11). In addition, recently Shan et al. (12) have demonstrated that browning of white adipose tissue in the MSTN-/ - mice is not a direct effect of MSTN deletion in the white adipose tissue, but an indirect effect from irisin that is regulated by MSTN and its downstream signaling cascade (AMPK-PGC1 $\alpha-$ FNDC5). In the same study, it was shown that treatment of cultured myotubes with anti-MSTN antibody or FST causes increased expression of both PGC1 $\alpha$ and FNDC 5 at the mRNA level, suggesting that these molecules might share common underlying mechanisms of action (12). Based on the above, we would expect to find a negative association between MSTN and irisin. Although we successfully demonstrated the expected negative association between MSTN and FST, we did not demonstrate any association between irisin and MSTN. This finding should be interpreted with caution taking into consideration that MSTN primarily functions in a paracrine/autocrine fashion and therefore associations at this level cannot be excluded. Alternatively, it is also possible that in vivo irisin is not influenced solely by MSTN levels but probably by the balance of the MSTNFST-activin system. Further large and powerful observational studies as well as mechanistic studies involving the interplay of all molecules involved in MSTN regulation and action need to be performed.

This is the first time that these myokines, irisin, FST, activin A, and MSTN, were studied in a cross-sectional study to elucidate the physiology of the FST-activin A-MSTN-irisin axis. We studied these associations in circulation and tissue level processing samples from young males and morbidly obese males and females. The existence of these associations cannot prove causality due to the cross-sectional design of the study and thus, we can only raise hypotheses to be tested by future larger observational as well as future mechanistic studies.

In conclusion, we report the existence of a positive association between FST and the newly identified myokine irisin. Follow-up studies would be helpful to confirm and expand our observations.

\section{Supplementary data}

This is linked to the online version of the paper at http://dx.doi.org/ 10.1530/EJE-13-0276.

\section{Declaration of interest}

The authors declare that there is no conflict of interest that could be perceived as prejudicing the impartiality of the research reported.

\section{Funding}

The Mantzoros Laboratory is supported by the National Institute of Diabetes and Digestive and Kidney Diseases (grants DK058785.
DK079929 and DK081913), and the National Institute on Aging (grant AG032030); as well as Award Number 1I01CX000422-01A1 from the Clinical Science Research and Development Service of the VA Office of Research and Development. The Cyprus study was supported by the Cyprus Research Promotion Foundation grant number EPYE $\Xi / 0205 / 10$.

\section{Author contribution statement}

M T Vamvini collected data and performed experiments, K N Aronis performed statistical evaluations, G Panagiotou, J P Chamberland, and J Y Huh assisted with experiments, M T Brinkoetter, M Petrou, C A Christophi, S N Kales, and D C Christiani collected data. C S Mantzoros designed the study and oversaw its performance. All authors have contributed to writing the manuscript.

\section{References}

1 Welc SS \& Clanton TL. The regulation of interleukin-6 implicates skeletal muscle as an integrative stress sensor and endocrine organ. Experimental Physiology 201398 359-371. (doi:10.1113/ expphysiol.2012.068189)

2 Henriksen T, Green C \& Pedersen BK. Myokines in myogenesis and health. Recent Patents on Biotechnology $2012 \quad 6 \quad 167-171$. (doi:10.2174/1872208311206030167)

3 Bostrom P, Wu J, Jedrychowski MP, Korde A, Ye L, Lo JC, Rasbach KA, Bostrom EA, Choi JH, Long JZ et al. A PGC1- $\alpha-$ dependent myokine that drives brown-fat-like development of white fat and thermogenesis. Nature $2012 \mathbf{4 8 1} 463-468$. (doi:10.1038/nature10777)

4 Sanchis-Gomar F, Lippi G, Mayero S, Perez-Quilis C \& GarciaGimenez JL. Irisin: a new potential hormonal target for the treatment of obesity and type 2 diabetes. Journal of Diabetes 20124 196. (doi:10.1111/j.1753-0407.2012.00194.x)

5 Huh JY, Panagiotou G, Mougios V, Brinkoetter M, Vamvini MT, Schneider BE \& Mantzoros CS. FNDC5 and irisin in humans: I. Predictors of circulating concentrations in serum and plasma and II. mRNA expression and circulating concentrations in response to weight loss and exercise. Metabolism 201261 1725-1738. (doi:10.1016/j.metabol.2012.09.002)

6 Choi YK, Kim MK, Bae KH, Seo HA, Jeong JY, Lee WK, Kim JG, Lee IK \& Park KG. Serum irisin levels in new-onset type 2 diabetes. Diabetes Research and Clinical Practice 2013100 96-101. (doi:10.1016/j.diabres.2013.01.007)

7 Liu JJ, Wong MD, Toy WC, Tan CS, Liu S, Ng XW, Tavintharan S, Sum CF \& Lim SC. Lower circulating irisin is associated with type 2 diabetes mellitus. Journal of Diabetes and its Complications 201327 365-369. (doi:10.1016/j.jdiacomp.2013.03.002)

8 Stengel A, Hofmann T, Goebel-Stengel M, Elbelt U, Kobelt P \& Klapp BF. Circulating levels of irisin in patients with anorexia nervosa and different stages of obesity - correlation with body mass index. Peptides 2013 39C 125-130. (doi:10.1016/j.peptides. 2012.11.014)

9 Kelly DP. Medicine. Irisin, light my fire. Science 2012336 42-43. (doi:10.1126/science.1221688)

10 Hansen J, Brandt C, Nielsen AR, Hojman P, Whitham M, Febbraio MA, Pedersen BK \& Plomgaard P. Exercise induces a marked increase in plasma follistatin: evidence that follistatin is a contraction-induced hepatokine. Endocrinology $2011 \mathbf{1 5 2}$ 164-171. (doi:10.1210/en.2010-0868)

11 Hittel DS, Axelson M, Sarna N, Shearer J, Huffman KM \& Kraus WE. Myostatin decreases with aerobic exercise and associates with insulin resistance. Medicine and Science in Sports and Exercise 2010 42 2023-2029. (doi:10.1249/MSS. Ob013e3181e0b9a8)

12 Shan T, Liang X, Bi P \& Kuang S. Myostatin knockout drives browning of white adipose tissue through activating the AMPK-PGC1 $\alpha$-Fndc 5 pathway in muscle. FASEB Journal 2013 27 1981-1989. (doi:10.1096/fj.12-225755) 
13 Vamvini MT, Aronis KN, Chamberland JP \& Mantzoros CS. Energy deprivation alters in a leptin- and cortisol-independent manner circulating levels of activin A and follistatin but not myostatin in healthy males. Journal of Clinical Endocrinology and Metabolism 201196 3416-3423. (doi:10.1210/jc.2011-1665)

14 Lee SJ, Lee YS, Zimmers TA, Soleimani A, Matzuk MM, Tsuchida K, Cohn RD \& Barton ER. Regulation of muscle mass by follistatin and activins. Molecular Endocrinology $2010 \quad 24 \quad 1998-2008$. (doi:10.1210/me.2010-0127)

15 Argiles JM, Orpi M, Busquets S \& Lopez-Soriano FJ. Myostatin: more than just a regulator of muscle mass. Drug Discovery Today 201217 702-709. (doi:10.1016/j.drudis.2012.02.001)

16 Hamnvik OP, Liu X, Petrou M, Gong H, Chamberland JP, Kim EH, Christophi CA, Kales SN, Christiani DC \& Mantzoros CS. Soluble leptin receptor and leptin are associated with baseline adiposity and metabolic risk factors, and predict adiposity, metabolic syndrome, and glucose levels at 2-year follow-up: the Cyprus Metabolism Prospective Cohort Study. Metabolism 201160 987-993. (doi:10.1016/j.metabol.2010.09.009)

17 Brinkoetter M, Magkos F, Vamvini M \& Mantzoros CS. Leptin treatment reduces body fat but does not affect lean body mass or the myostatin-follistatin-activin axis in lean hypoleptinemic women. American Journal of Physiology. Endocrinology and Metabolism 2011301 E99-E104. (doi:10.1152/ajpendo.00146.2011)

18 Pedersen BK \& Febbraio MA. Muscles, exercise and obesity: skeletal muscle as a secretory organ. Nature Reviews. Endocrinology 20128 457-465. (doi:10.1038/nrendo.2012.49)

19 Roberts MD, Bayless DS, Company JM, Jenkins NT, Padilla J, Childs TE, Martin JS, Dalbo VJ, Booth FW, Rector RS et al. Elevated skeletal muscle irisin precursor FNDC5 mRNA in obese OLETF rats. Metabolism 201362 1052-1056. (doi:10.1016/j.metabol. 2013.02.002)

20 Swick AG, Orena S \& O'Connor A. Irisin levels correlate with energy expenditure in a subgroup of humans with energy expenditure greater than predicted by fat free mass. Metabolism 201362 1070-1073. (doi:10.1016/j.metabol.2013.02.012)

21 Fain JN, Company JM, Booth FW, Laughlin MH, Padilla J, Jenkins NT, Bahouth SW \& Sacks HS. Exercise training does not increase muscle FNDC5 protein or mRNA expression in pigs. Metabolism 201362 1503-1511. (doi:10.1016/j.metabol.2013. 05.021)

22 Moon HS, Dincer F \& Mantzoros CS. Pharmacological concentrations of irisin increase cell proliferation without influencing markers of neurite outgrowth and synaptogenesis in mouse H19-7 hippocampal cell lines. Metabolism 201362 1131-1136. (doi:10.1016/j.metabol.2013.04.007)

23 Polyzos SA, Kountouras J, Shields K \& Mantzoros CS. Irisin: a renaissance in metabolism? Metabolism 201362 1037-1044. (doi:10.1016/j.metabol.2013.04.008)

24 de Kretser DM, Hedger MP, Loveland KL \& Phillips DJ. Inhibins, activins and follistatin in reproduction. Human Reproduction Update 20028 529-541. (doi:10.1093/humupd/8.6.529)

25 Nakatani M, Kokubo M, Ohsawa Y, Sunada Y \& Tsuchida K. Follistatin-derived peptide expression in muscle decreases adipose tissue mass and prevents hepatic steatosis. American Journal of Physiology. Endocrinology and Metabolism 2011300 E543-E553. (doi:10.1152/ajpendo.00430.2010)

26 Moreno-Navarrete JM, Ortega F, Serrano M, Guerra E, Pardo G, Tinahones F, Ricart W \& Fernandez-Real JM. Irisin is expressed and produced by human muscle and adipose tissue in association with obesity and insulin resistance. Journal of Clinical Endocrinology and Metabolism 201398 E769-E778. (doi:10.1210/jc.2012-2749)

27 Roca-Rivada A, Castelao C, Senin LL, Landrove MO, Baltar J, Belen Crujeiras A, Seoane LM, Casanueva FF \& Pardo M. FNDC5/irisin is not only a myokine but also an adipokine. PLoS ONE 20138 e60563. (doi:10.1371/journal.pone.0060563)

28 McPherron AC. Metabolic functions of myostatin and Gdf11. Immunology, Endocrine $\mathcal{E}$ Metabolic Agents in Medicinal Chemistry 201010 217-231. (doi:10.2174/187152210793663810)

29 Dani C. Activins in adipogenesis and obesity. International Journal of Obesity 201337 163-166. (doi:10.1038/ijo.2012.28)

Received 2 April 2013

Revised version received 30 July 2013

Accepted 23 September 2013 